\title{
EDITORIAL
}

\author{
Koji Koyamada $\cdot$ Xiaoru Yuan
}

\section{The Visualization Workshop 2013}

Published online: 5 August 2014

(c) The Visualization Society of Japan 2014

\section{Guest Editors}
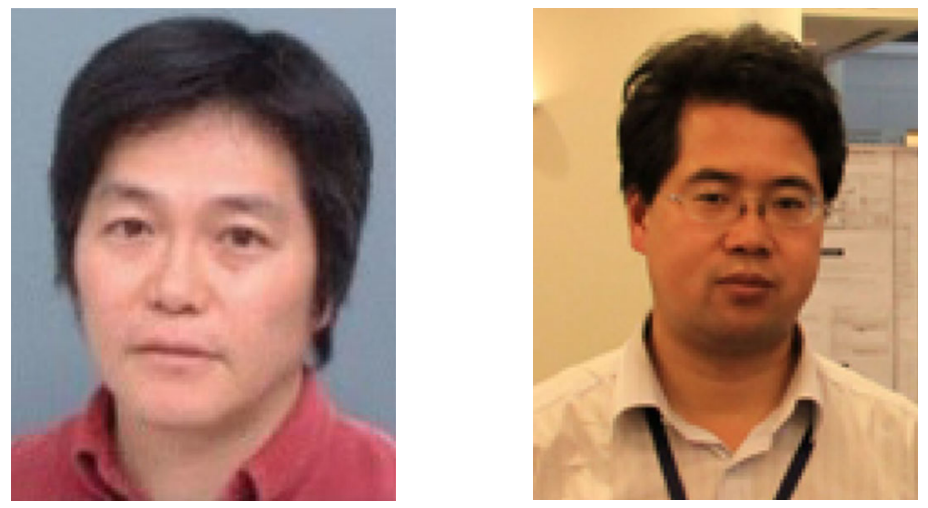

The Visualization Workshop 2013 was held in Beijing, China, on 12-13 July 2013. The number of participants was over 510 from China and a few other counties. The state of arts in many aspects of scientific visualization, information visualization and visual analytics was presented and discussed.

The workshop invited three keynotes, including topics of big data visualization with graph and map by Dr. Yifan Hu from AT\&A labs, urban data visualization by Prof. Claudio Silva from NYU, and visualization for masses in the big data era by Prof. Huamin Qu from HKUST. The workshop also invited more than 20 talks, covering research topics of network visualization, traffic visualization, large-scale scientific visualization, text visualization, visualization from journalism, etc.

In the workshop, 32 submissions are selected for presentation in the conference. Among those presentations, four papers were further selected by the Program Committee of Visualization Workshop 2013. After the standard peer review process of Journal of Visualization (JOV), those four papers were finally accepted for publication.

We wish to thank the editors and reviewers of JOV for making it possible to publish this special issue from the Visualization Workshop 2013. We also thank the authors for their careful and insightful work and

K. Koyamada

Kyoto University, Kyoto, Japan

X. Yuan $(\square)$

Peking University, Beijing, China

E-mail: xiaoru.yuan@pku.edu.cn 
cooperation in the preparation of the revised papers. It will be our pleasure if readers appreciate the hot topics in general visualization research as a result of this special issue. In 2014, the Visualization Workshop will be transformed to Visual Analytics Conference. We would like to express our sincere thanks to the staff at Springer Verlag for their kind support. 